\title{
Enhancement of Pool Boiling Heat Transfer Performance by an Eco- Friendly Surfactant
}

\author{
Rinku Kumar Gouda ${ }^{1}$, Manabendra Pathak ${ }^{1 *}$, Mohd. Kaleem Khan ${ }^{1}$ \\ ${ }^{1}$ Indian Institute of Technology Patna \\ Bihta, Patna, Bihar-801103, India. \\ rinku.pme15@iitp.ac.in; *mpathak@iitp.ac.in; mkkhan@iitp.ac.in
}

\section{Extended Abstract}

Heat transfer through pool boiling is a key process to many industrial and household applications like thermal power plant, nuclear reactor, etc. It involves large rate of heat transfer with less temperature difference. However, heat transfer coefficient in pool boiling on smooth surface is not adequate to dissipate large amount of heat required for various applications. Therefore, several passive heat transfer enhancement techniques have been proposed like heater surface modification [1] or additive addition in base fluid [2] etc. Additive addition to base fluid such as surfactant has gain popularity because it involves high heat transfer while maintaining low wall superheat. Further in surfactant addition, its concentration is low enough when added in working fluid. For surfactant, except properties like viscosity and surface tension the other properties like density, saturation temperature etc. remains unaffected [2]. Therefore, it is important to estimate the maximum concentration (critical micelles concentration: $\mathrm{CMC}$ ) to be added in working fluid which prevent the formation of micelles. At CMC, surface tension of aqueous surfactant is minimum. Reduction in surface tension helps in enhancing the heat transfer rate in pool boiling due to enhanced nucleation sites and absence of bubble coalescence.

Several studies have reported heat transfer enhancement in pool boiling using different types of surfactants [2]. Although addition of these surfactants in working fluid helps in increasing heat transfer rate, but most of these surfactants are not suitable for industrial purpose because of their toxicity to aquatic organisms and non-biodegradable in nature. So, it is necessary to search for a new surfactant with insignificant environment impact.

The objective of the current study is to explore the performance of a new biodegradable surfactant i.e. Rhamnolipid solution for heat transfer enhancement in pool boiling. Rhamnolipid is produced from microorganisms and is biodegradable in nature. In the present work experimental investigation of pool boiling with aqueous Rhamnolipid surfactant at 4 different concentrations (100 ppm, $200 \mathrm{ppm}, 300 \mathrm{ppm}$ and $400 \mathrm{ppm}$ ) have been carried out on smooth surface and its heat transfer characteristics have been compared with pure water. The heater surface was fabricated on a copper substrate with a foot print area of $10 \times 10 \mathrm{~mm}^{2}$. Prior to any experiment the prepared solution was first magnetically stirred for $1 \mathrm{hr}$. CMC value was calculated based on surface tension values of the solution measured by Force Tensiometer instrument. The CMC value was found to be $200 \mathrm{ppm}$. Pool boiling study was carried out at atmospheric condition for different heat fluxes. It was observed that addition of Rhamnolipid surfactant to pure water reduces the surface tension by nearly $50 \%$ to that of pure water and increases the heat transfer rate. On increasing concentration, the boiling curve shifts left ward relative to that of pure water. Optimum boiling performance was observed at CMC and afterwards no enhancement was observed. Compared to pure water, an enhancement of $101.22 \%$ in heat transfer coefficient was observed in surfactant solution at CMC. Enhancement in heat transfer may be attributed to reduced surface tension of surfactant fluid. Reduction in surface tension increases the active nucleation site density and also the bubble departure frequency.The surfactant additive Rhamnolipid can be successfully used to enhance the heat transfer rate in pool boiling.

Keywords: Pool boiling, heat transfer coefficient, surfactant, biodegradable, Rhamnolipid.

\section{References}

[1] R. K. Gouda, M. Pathak, M. K. Khan, "Pool boiling heat transfer enhancement with segmented finned microchannels structured surface," Int. J. Heat Mass Transfer., vol. 127, pp. 39-50, 2018.

[2] R. K. Gouda, M. Pathak, M. K. Khan, "A biosurfactant as prospective additive for pool boiling heat transfer enhancement," Int. J. Heat Mass Transfer., vol. 150, pp. 119292, 2020. 